\title{
Evaluation of Emerging Antimicrobials Resistance in nosocomial infections caused by E. coli: The comparison results of observed cases and compartmental model
}

mohammad kogani

Iran University of Medical Sciences: Tehran University of Medical Sciences

babak eshrati

Iran University of Medical Sciences: Tehran University of Medical Sciences

hamid reza baradaran

Iran University of Medical Sciences: Tehran University of Medical Sciences

leila janani

Iran University of Medical Sciences: Tehran University of Medical Sciences

mahshid nasehii ( $\triangle$ mah.nasehii@gmail.com )

IUMS: Iran University of Medical Sciences https://orcid.org/0000-0003-4283-3441

\section{Research}

Keywords: Antimicrobials Resistance, Emerging Antimicrobial Resistance, E.coli, Nosocomial Infections, Compartmental Model

Posted Date: May 14th, 2021

DOI: https://doi.org/10.21203/rs.3.rs-495402/v1

License: (c) (i) This work is licensed under a Creative Commons Attribution 4.0 International License.

Read Full License 


\title{
Evaluation of Emerging Antimicrobials Resistance in nosocomial infections caused by E. coli: The comparison results of observed cases and compartmental model
}

\begin{abstract}
Background: Early detection of Antimicrobials Resistance outbreaks is one of the most important goals of the World Health Organization. In this study, by comparing the observed cases of resistance with its expected cases, the outbreak of these resistances was investigated. It should be noted that this subject was not done in the country until the time of the study.

Methods: This study is a hospital-based study. Data related to all the university general of Iran (57 hospitals) were used. In these hospitals, all the patients who were infected by E.coli in time period of March 212017 to March 202018 were enrolled in the study. Then, using an index called the SIR; the observed cases of resistant E.coli were compared with the expected ones. This index is achieved from dividing the observed cases by its expected cases. If the obtained number is greater than one, it indicates the greater observed cases rather than the expected cases, which can represent Emerging. In order to compute SIR index, we divided the number of observed cases of Antimicrobials Resistance E.coli by the number of expected cases of each Antimicrobials Resistance E coli. To predict the expected cases of each Antimicrobials Resistance E coli, we developed one compartmental model. In this model, the number of patients is estimated using equations. Berkeley Madonna version 8.3.23 software was used to manipulate these equations.
\end{abstract}

Results: The SIR index for E.coli resistant to Ampicillin, Ceftazidime and Colistin were 1.2(1.1-1.3), 1.1(1.02-1.2) and 1.7(1.02-2.3) respectively. This index for E.coli resistant to Meropenem was .8 (.6-.9). In other cases, the calculated index was not statistically significant.

Conclusions: Ampicillin-resistant E.coli and Ceftazidime-resistant E.coli observed cases among nosocomial infections were greater than the expected cases. Hence it is necessary to reconsider using such type of antibiotics in treatment of nosocomial infections caused by E.coli. The results of this study could be important for health policy makers. In the future, outbreaks of this type of infection can be investigated with the help of the results of this study.

Keywords: Antimicrobials Resistance; Emerging Antimicrobial Resistance; E.coli; Nosocomial Infections; Compartmental Model 


\section{Background}

World Health Organization (WHO) emphasized repeatedly on spread and association of nosocomial infections with antimicrobial resistance (AMR) and introduced it as a critical risk for public health that needs specific interventions. At 2014, WHO named AMR as a global threat on a report, which due to the importance of Antimicrobials Resistance (ABR) and insufficiency of necessary knowledge and attempts to face it the main concentration of the report was on this health concern. This report stated that in many regions of the world, treatment with antibiotic for simple infections has no effect anymore. In addition, systematic reviews in this regard showed that such types of resistance can have adverse effects as the consequence of treatment(1).

AMR in recent years becomes rapidly a health concern in most health care settings(2). Antimicrobials Resistance infections are seen commonly due to high accumulation of at risk patients and high rate of using antibiotics in hospitals. Such infections increase medical costs so that the estimates show increase among 19-29 \$ per case(3). In all regions of WHO, high percentage of bacteria which cause nosocomial infections are treatment-resistant. The most common bacterial factor among the nosocomial infections is E.coli (1). Urinary tract infection is among the most common nosocomial hospitals which E.coli is the most common bacterial factor (4-6).

In recent years, the world was faced to Antimicrobials Resistance E.coli in most regions (57) which seriously threaten public health and increases therapeutic costs and additional costs (8). Percentage of third generation cephalosporin-and fluoroquinolones resistance E.coli in EMRO region was among 21 to 63, and in Iran for third generation cephalosporin was $41 \%$ and for fluoroquinolones was $54 \%$ (1).

Action plan of AMR was developed at the $68^{\text {th }}$ meeting of the WHO at 2015 (9). One of the strategic aims of this action plan is reinforcement of evidence base through increase in global care of AMR and increase of research in this field. The WHO developed the Global Antimicrobial Resistance Surveillance System (GLASS) program in regard with this strategic objective (10). In GLASS program, samples are collected from the patients with nosocomial infection through microbiological methods in order to diagnose AMR, which its pathogenic type and its resistance or sensitivity against the antibiotics are identified through performing antimicrobial susceptibility testing (AST) (10).

The laboratory method of Disk-Diffusion is used in nosocomial infections care system of Iran to diagnose Antimicrobials Resistance which with other information related to nosocomial infections gathered from the hospitals in Iranian Nosocomial Infections Surveillance Software - INIS are recorded (11). 
According to the GLASS program, in E.coli pathogen, data related to seven following classes of antibiotic are collected: Sulfonamides and Trimethoprim, Fluoroquinolones, Thirdgeneration cephalosporins, Fourth-generation Cephalosporins, Carbapenems, Polymyxins, and Penicillins. For each of these mentioned antibiotic classes, antibiotic factor which might be used for AST is as follow: Co-trimoxazole, Ciprofloxacin or levofloxacin, Ceftriaxone or Cefotaxime and Ceftazidime, Cefepime, Imipenem and Meropenem, Colistin, Ampicillin.

According to the National Nosocomial Infection Surveillance System (NNIS), in those with nosocomial infection caused by E.coli, AST must also be used for at least one of the eleven antibiotics mentioned above.

One of the most important objectives of the GLASS program is well-timed detection and transmission of Emerging Antimicrobial Resistance (EAR). In the context of AMR, one of the main ways of detection of Emerging in national and local level is identification of deviations from the expected resistance in pharmaceutical compounds (10). Subsequently, it can be said that if observed cases of one ABR is higher than its expected cases, it can be an indication of Emerging.

Regarding nosocomial infections, CDC uses an index named Standardized Infection Ratio (SIR) (12). This index is achieved from dividing the observed cases of infection by its expected cases. If the obtained number is greater than one, it indicates the greater observed cases rather than the expected cases, which can represent Emerging, and if this index is less than one, it can be the result of underreporting (12). So far, regression models have been used to estimate the expected cases. Regression models require many variables, which make the task difficult. But if you use compartment models, this problem does not exist. These models are also easier to use for hospitals.

In line with the GLASS program, national nosocomial infections system (NNIS) in IRAN (11) was integrated with AMR program, and was implemented at hospitals of the country. Now due to importance of the E.coli in nosocomial infections, Emerging Antimicrobial Resistance (EAR) in E.coli can be assessed using SIR index. It should be noted that this subject was not done in the country divided by mentioned antibiotics until the time of the study.

Subsequently, the main aim of the current study is to assess EAR using an index named SIR among the mentioned antibiotics of the GLASS program (10), which are used in treatment of nosocomial infections caused by E.coli microbe.

\section{Methods}

This hospital-based study was operated in compliance with the Strengthening the Reporting of Observational Studies in Epidemiology statement (STROBE statement)(13) and Transparent Reporting of a multivariable prediction model for Individual Prognosis Or 
Diagnosis (TRIPOD)(14). Ethical approval for this study was provided by the Ethic Committee of IRAN University of Medical Sciences (No.IR.IUMS.REC.1398.211).

In this study, part of the data of NNIS (11) has been used. In this study, data related to all the university general or referral hospitals at the capitals of the provinces of Iran (57 hospitals) were used. In these hospitals, all the patients who were infected by E.coli in time period of March 212017 to March 20 2018, and were examined regarding resistance for at least one of these the 11 antibiotic factors, were enrolled in the study.

In addition, due to the hypotheses present in discussion of model and incidence rate, it is more explained as follow, the cases that the duration between hospitalization and incidence of nosocomial infection of them was in normal range (quartile1 - quartile3) and/or less than 25 percentile, were remained in study and we assessed them separately.

Finally, in this study, the results of the 12954 tests of antibiogram were used (Table 1), result of each of the tests showed the nosocomial infection occurred is resistant to which of the mentioned 11 antibiotics.

Data related to these cases were divided in two sections due to the reasons explained below (Table 1):

1- First part: data related to first half of the year was done by 5701 tests.

2- Second part: data related to second half of the year was done by 7253 tests.

In this study, we aimed to investigate EAR in E.coli, divided by the mentioned antibiotics. Therefore, our primary outcome was having or not having antibiotic resistant E.coli, which indicates nosocomial infection caused by E.coli which shows resistance to the mentioned antibiotics with the Disk-Diffusion test. In order to assess EAR, we used an index named standardized infection ratio (SIR) (12). In order to compute this index, we divided the number of observed cases of Antimicrobials Resistance E coli by the number of expected cases of each Antimicrobials Resistance E coli.

If the confidence interval involved the number 1 indicates the non-significance, in other case and if it does not involve number 1 , indicates significance.

In this study, to predict the expected cases of each Antimicrobials Resistance E coli, we developed one compartmental model (15). The populations in such models are classified in sub-groups (compartment) which produce the model's structure in fact. Model structure is selected due to the aim of the study and natural history of the infection (15). We used SIR structure for the compartmental model due to the aim of the study, which is the expected prediction of each Antimicrobials Resistance E coli at a definite time.

In this structure, study population was classified in three compartment or subgroup into susceptible (S), infectious (I), and remove (R). Susceptible individuals are the cases who were nosocomial infected, infectious individuals are those with nosocomial infections with resistance, and the remove ones are the infectious individuals who are discharged due to 
any reason (death, treated). The model describes the infection transmission using the total number of individuals in these compartments.

Since in this study, estimating the number of cases is considered (not the probability), hence, we used difference equations to set up the compartmental model. In this approach, translocation of the cases between sub-groups is described using discrete time steps (such as day). In fact, this method is to estimate the number of cases in a definite time (15). To manipulate difference equations in this study, Euler method in the package of Berkeley Madonna version 8.3.23 was used. This method is applied specifically for setting up difference equations (15). Berkeley Madonna package enables us to set up the model in a simpler way through drawing a diagram (15).

In fact, through this method, instead that the difference equations will be typed manually, the model was constructed graphically, and subsequently, the equations of the model are also prepared in text. In this study, we also developed the model separately for each of the eleven antibiotics by drawing the diagram of the model (figure 1).

Figure 1: developed compartmental model diagram

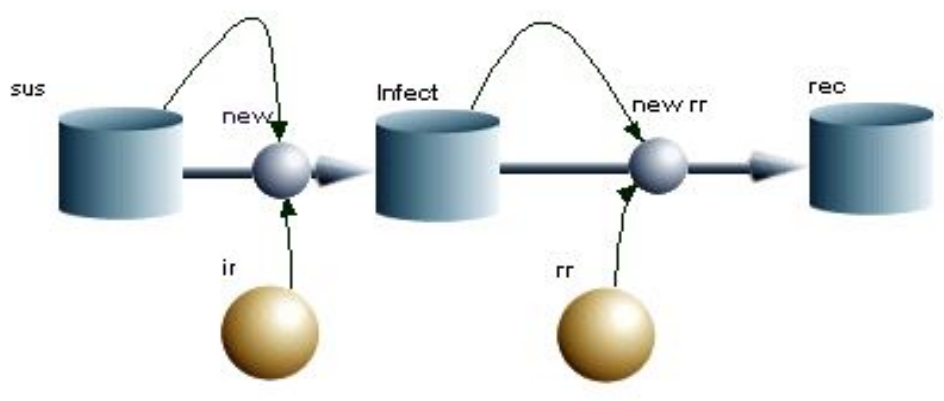

- sus: susceptible: total number of cases with nosocomial infection caused by E.coli

- ir: incidence rate of Antimicrobials Resistance E.coli per each day

- New: number of cases who developed Antimicrobials Resistance E coli in duration of $t$ and $t+1 .($ ir $\times$ sus $)$

- Infect: number of cases with Antimicrobials Resistance E coli in the time of $t$

- rr: ratio of Antimicrobials Resistance E coli cases removed from hospital per each day

- New rr: cases of Antimicrobials Resistance E.coli who are removed among the time of $t$ and $t+1 .(r r \times$ infect $)$

- rec: number of cases with Antimicrobials Resistance E coli removed at time $t$

Model difference equations produced is presented below: 


$$
\begin{aligned}
& \operatorname{sus}(t+d t)=s u s-n e w \\
& \text { infect }(t+d t)=i n f e c t+n e w-n e w_{-} r r \\
& r e c(t+d t)=r e c+n e w_{-} r r
\end{aligned}
$$

- $\operatorname{sus}(t+d t)$ : number of cases of nosocomial infection caused by E.coli at time of $t+1$

- infect $(t+d t)$ : number of Antimicrobials Resistance E coli at time of $t+1$

- $\operatorname{rec}(t+d t)$ : number of Antimicrobials Resistance $\mathrm{E}$ coli cases removed at time $t+1$

- $\mathrm{t}$ : times used in the model (times between start time and stop time)

- dt: size of time step in daily units (one day)

When just one dataset is available, we can non-randomly divide it into two sections, develop the model in one section of it, and assess its performance in another part (14). Therefore, in this study, we divided data into two sections (data related to the first half of year and data related to the second half of the year), which we run the developed model in the second part of the data to predict the cases expected for resistance.

We needed several unknown parameters (incidence rate (ir), remove rate ( $\mathrm{rr}$ ), and appropriate time gap) during model developing. Since in the literature review we performed, there were no study with setting similar to ours study, which these parameters could be extracted from it; therefore, we extracted these parameters from the first part of data (table 3, 4 and 5).

We assessed the validity and performance of the model through running the model in first part of data prior to perform the developed model for each 11 antibiotics in the second part of data to predict the Antimicrobials Resistance E coli expected cases, which in case of necessity (difference among predicts of the model and reality) the model was adjusted (optimized) (table 4 and 5). We summed all the new cases of Antimicrobials Resistance which the model predicted among start time and stop time to be able to estimate all the predicted cases of each Antimicrobials Resistance. An additional file shows this in more detail [see Additional file 1].

One important hypothesis in estimating the incidence rate based on individual-year is remaining fixed (approximately) of risk event over the time (16). In addition, the hypothesis present in models of infectious diseases is that individuals become sick at a constant rate (15). 
Therefore, we must select the time period so that our required time duration was a correct average, hence, we calculated the mean and median of the variable "hospitalization until event" based on each antibiotic in the first section of data, and since in all the antibiotics, mean and median was considerably different and indicates non-normal distribution of the variable (table 2), therefore, we used participants who were among the quartile1 quartile3 of the variable of "hospitalization until event", or were lower than quartile1 (table 1). Distribution of the variable of "hospitalization until event" was normal within these two gaps. By the way, required and appropriate time gap (start time-stop time) to run the compartmental model was estimated, and the ir extracted was the ir which includes the above stated hypothesis.

As stated, there is mostly a hypothesis in infectious diseases models which states that individuals become sick at a constant rate, and when they get sick, they will recover at a constant rate, therefore, rate in such cases can be calculated through dividing the number 1 by "average time for the event" (15). In this study, we also computed average time for that a resistant individual removed from the hospital (for death or discharge) to compute remove rate ( $\mathrm{rr}$ ) parameter divided by each antibiotic, and we estimated this parameter by dividing the number 1 by it (table 3 ).

Table 1: number of performed antibiogram tests divided by the antibiotic in normal range of data

\begin{tabular}{|l|l|l|l|l|l|l|}
\hline \multirow{2}{*}{$\begin{array}{l}\text { Antimicrobial } \\
\text { agents }\end{array}$} & $\begin{array}{l}\text { Number of all the } \\
\text { performed tests }\end{array}$ & \multicolumn{2}{l}{$\begin{array}{l}\text { Number of performed test } \\
\text { in first section** }\end{array}$} & $\begin{array}{l}\text { Number of performed test } \\
\text { in second section*** }\end{array}$ \\
\cline { 2 - 7 } & $\begin{array}{l}\text { Q1-Q3 } \\
\text { among }\end{array}$ & $<$ Q1 & $\begin{array}{l}\text { Q1-Q3 } \\
\text { among }\end{array}$ & $<$ Q1 & $\begin{array}{l}\text { Q1-Q3 } \\
\text { among }\end{array}$ & $<$ Q1 \\
\hline Ampicillin (AMP) & 400 & 143 & 185 & 56 & 215 & 87 \\
\hline Ceftazidime (CAZ) & 949 & 391 & 381 & 150 & 568 & 241 \\
\hline Ceftriaxone (CEF) & 1073 & 469 & 498 & 213 & 575 & 256 \\
\hline Cefotaxime (CTX) & 889 & 366 & 364 & 158 & 525 & 208 \\
\hline Cefepime (CFP) & 644 & 239 & 306 & 114 & 338 & 125 \\
\hline Ciprofloxacin (CIP) & 1516 & 755 & 714 & 342 & 802 & 413 \\
\hline Levofloxacin (LvX) & 195 & 72 & 73 & 31 & 122 & 41 \\
\hline Colistin (CST) & 238 & 107 & 87 & 29 & 151 & 78 \\
\hline Imipenem (IPM) & 1324 & 564 & 563 & 223 & 761 & 341 \\
\hline Meropenem (MEM) & 708 & 264 & 319 & 108 & 389 & 156 \\
\hline Cotrimoxazole(SXT) & 1108 & 540 & 530 & 257 & 578 & 283 \\
\hline TOT & 9044 & 3910 & 4020 & 1681 & 5024 & 2229 \\
\hline
\end{tabular}


* Total number of performed tests during year (study subjects).

** Number of tests performed in the first half of the year 2017 (required subjects to assess model performance)

*** Number of tests performed in the second half of the year (subjects required to perform the model and assess emerging)

Table 2: range, mean, median and appropriate estimated time gap for the variable "hospitalization until event", in the first section of data, divided by antibiotic

\begin{tabular}{|c|c|c|c|c|c|}
\hline \multirow[t]{2}{*}{$\begin{array}{l}\text { Antimicrobial } \\
\text { agents }\end{array}$} & \multirow[t]{2}{*}{ Range } & \multirow[t]{2}{*}{$\begin{array}{l}\text { Mean } \\
\text { (SD) }\end{array}$} & \multirow[t]{2}{*}{$\begin{array}{l}\text { Median } \\
\text { (Q1-Q3) }\end{array}$} & \multicolumn{2}{|c|}{ Appropriate and required time gap } \\
\hline & & & & In (Q1-Q3) & in $\mathrm{Q} 1>$ \\
\hline Ampicillin (AMP) & $3-377$ & $20(36)$ & $11(5-20)$ & $5-20$ & $3-4$ \\
\hline Ceftazidime (CAZ) & $3-430$ & $20(45)$ & $9(5-18)$ & $5-18$ & $3-4$ \\
\hline Ceftriaxone (CEF) & $3-429$ & 19(41) & $9(5-18)$ & $5-18$ & $3-4$ \\
\hline Cefotaxime (CTX) & $3-430$ & $21(47)$ & $9(5-18)$ & $5-18$ & $3-4$ \\
\hline Cefepime (CFP) & $3-429$ & $17(29)$ & $10(5-20)$ & $5-20$ & $3-4$ \\
\hline Ciprofloxacin (CIP) & $3-430$ & 19(44) & $8(5-17)$ & $5-17$ & $3-4$ \\
\hline Levofloxacin (Lvx) & $3-369$ & $17(35)$ & $9(4-17)$ & $4-17$ & $3-3$ \\
\hline Colistin (CST) & $3-371$ & $20(42)$ & $11(6-18)$ & $6-18$ & $3-5$ \\
\hline Imipenem (IPM) & $3-430$ & $21(50)$ & $9(5-19)$ & $5-19$ & $3-4$ \\
\hline Meropenem (MEM) & $3-429$ & $18(35)$ & $10(5-19)$ & $5-19$ & $3-4$ \\
\hline Cotrimoxazole(SXT) & $3-385$ & $18(39)$ & $8(5-17)$ & $5-17$ & $3-4$ \\
\hline
\end{tabular}


Table 3: estimated range, mean and median for variable of "event-discharge" in first section of data, divided by antibiotic

\begin{tabular}{|c|c|c|c|c|c|}
\hline $\begin{array}{l}\text { Antimicrobial } \\
\text { agents }\end{array}$ & Range & Mean (SD) & Median (Q1-Q3) & $\begin{array}{c}\text { Mean }^{a}(\mathrm{SE}) \\
\text { In normal range }\end{array}$ & $\mathrm{rr}^{\mathrm{b}}$ \\
\hline Ampicillin (AMP) & $1-377$ & $18(30)$ & $10(5-19)$ & $10(.2)$ & $1 / 10=.1$ \\
\hline Ceftazidime (CAZ) & $1-377$ & $19(27)$ & $11(5-22)$ & $11(.2)$ & $1 / 11=.09$ \\
\hline Ceftriaxone (CEF) & $1-132$ & $15(17)$ & $10(4-20)$ & $10(.2)$ & $1 / 10=.1$ \\
\hline Cefotaxime (CTX) & $1-159$ & 17(19) & $11(5-22)$ & $11.7(.3)$ & $1 / 11.7=.09$ \\
\hline Cefepime (CFP) & $1-132$ & $17(18)$ & $12(5-22)$ & $12(.4)$ & $1 / 12=.08$ \\
\hline Ciprofloxacin (CIP) & $1-119$ & $15(16)$ & $10(4-20)$ & $10(.3)$ & $1 / 10=.1$ \\
\hline Levofloxacin (Lvx) & $1-97$ & 12(15) & $7(3-14.5)$ & $7(.5)$ & $1 / 7=.14$ \\
\hline Colistin (CST) & $2-71$ & $27(23)$ & $21(10.5-42)$ & 16(1.6) & $1 / 16=.06$ \\
\hline Imipenem (IPM) & $1-116$ & $16(16)$ & $12(5-23)$ & $12.4(.5)$ & $1 / 12.4=.08$ \\
\hline Meropenem (MEM) & $1-132$ & $16(17)$ & $10(4-21)$ & $10.7(.3)$ & $1 / 10.7=.09$ \\
\hline Cotrimoxazole(SXT) & $1-132$ & 15(17) & $9(3.5-19)$ & $9(.2)$ & $1 / 9=.11$ \\
\hline
\end{tabular}


a average time for that one resistant person is removed from hospital. This mean was achieved in the range of q1-q3 of "event-discharge".

b: remove rate based on each day.

\section{Model validation and optimization:}

Performance and validation of the developed model must be assessed prior to performing the data. This can be done by comparing outcomes of the developed model and observed data (13). We run the model in first section of data to assess performance of the model. We did this by comparing predicted resistance cases of the model with observed resistance cases (table 4 and 5).

If the predicted resistance cases were similar to the observed resistance cases and had no significant difference, indicates good performance of the model. In cases which the difference among them was statistically significant, we changed the parameter of ir until that least gap among the number of predicted cases of resistance and its observed cases occurred. We done this using a function named Optimize (13) that is existed in the package of Berkeley Madonna.

In such cases which available dataset is classified based on time into two sections, which the model is developed in one part of it and the performance is assessed in another part, the validation of the model is considered at moderate level among internal and external validity (14).

\section{Results}


Out of 5554 cases with nosocomial infection caused by E.coli, at least one antibiogram test was done for 4129 cases. Out of this number, 168 cases were dropped from the study due to operator's error in entering data related to date of hospitalization, which leads to occurring negative numbers in variable of hospitalization length. Therefore, at 2017, 3961 cases of nosocomial infection caused by E.coli were occurred who performed at least one antibiogram test. Out of this number, we involved 3059 patients who were located in normal range of hospitalization to infection. Out of these patients, 12954 tests were done to diagnose resistances which are presented in table 1 , divided by the first and second half of the year.

Mean of the hospitalization until nosocomial infection among the patients was 8 (SD=5) and median for them was 6 (4-11). For patients who were not in the normal range of data, mean was $52(\mathrm{SD}=61)$ and median was 34 (27-49).

Mean age of those who were assessed in the first half of the year (first part of data), and second half of the year (second part of data) was $56(\mathrm{SE}=0.6)$ and 58 ( $\mathrm{SE}=0.5)$, respectively. Fifty seven percent of those who were assessed in the first half of the year were man. Among those who were assessed in the second half, 54\% were man.

Table 2 shows the appropriate estimated time gap (start time-stop time) to develop the model divided by eleven antibiotics. This time gap is presented in two sections among Q1Q3 and lesser than the percentile 25 of the hospitalization time.

Range of "hospitalization to event", depended on type of antibiotic, is among 3-430 days. This range in time gap among Q1-Q3 is 4-20 days, based on the type of antibiotic. Mean of the "hospitalization to event" is among 17-21 days, based on the type of antibiotic. Median of "hospitalization to event" is among 8-11 days, based on type of antibiotic. In addition, in this time gap, remove rate and incidence rate were estimated which their values can be observed in tables 3 and 4, respectively.

Table 3 shows the time range, mean and median for variable of "event-discharge". This range, based on type of antibiotic, varies among 1-377 days. The mean for this variable varies among 12 days for Lvx to 27 days for CST. The median also varies among 7 days for Lvx to 21 days for CST.

Mean for variable of "event-discharge" within normal time range varies among 7 days for Lvx to 16 days for CST. By dividing number 1 by the mean, based on the antibiotic, rr was estimated. This rate varies among 0.06 for Colistin and 0.14 for Levofloxacin.

As table 6 showed, the model is developed for the two time ranges. Size of time step was considered as 1 day for all the antibiotics. The results of the performed model in first section of data are presented in table 4 and 5 to assess model performance.

In table 4, the outcome of the model (expected cases) for antibiotics of Ceftriaxone (CEF), Cefotaxime (CTX), and co-trimoxazole (SXT) significantly differed from the observed cases, 
which by optimizing the parameter of ir, those become similar as much as possible. In Table 5, the outcome of the model (expected cases) for antibiotics of Ceftriaxone (CEF), Cefotaxime (CTX), Ciprofloxacin and co-trimoxazole (SXT) significantly differed from the observed cases, which by optimizing the parameter of ir, those become similar as much as possible. You can observe the optimized incidence rate in this table. Finally, you can observe optimized developed model, divided by antibiotic, in table 7 .

You can observe in Table 8 the number of nosocomial infections caused by E.coli which was occurred in second section of data. Among these nosocomial infections, number of cases of resistance observed based on each antibiotic is also presented. In addition, number of cases of resistance which is predicted by the model is also presented. In this table, computed SIR index is also presented.

Table 4: Incidence rate and optimized incidence rate of Antimicrobials Resistance E.coli In normal time range

\begin{tabular}{|c|c|c|c|c|c|c|c|c|}
\hline $\begin{array}{l}\text { Antimicrobial } \\
\text { agents }\end{array}$ & sus & obs & $\begin{array}{c}\text { Number of person- } \\
\text { days of } \\
\text { hospitalization }\end{array}$ & $\begin{array}{c}\text { Incidence rate } \\
(95 \% \mathrm{CI})\end{array}$ & $\exp$ & $\begin{array}{l}\text { Significant } \\
\text { difference }\end{array}$ & Optimized ir & $\begin{array}{c}\text { Optimized } \\
\text { expected } \\
\text { cases } \\
\end{array}$ \\
\hline Ampicillin (AMP) & 185 & 150 & 1926 & $.08(.07-.09)$ & 135 & $\mathrm{~N}^{1}$ & - & - \\
\hline Ceftazidime (CAZ) & 381 & 247 & 3579 & $.07(.06-.08)$ & 243 & $\mathrm{~N}$ & - & - \\
\hline Ceftriaxone (CEF) & 498 & 381 & 4684 & $.08(.07-.09)$ & 343 & $\mathrm{Y}^{2}$ & .1 & 385 \\
\hline Cefotaxime (CTX) & 364 & 294 & 3596 & $.08(.07-.09)$ & 251 & $Y$ & .1 & 281 \\
\hline Cefepime (CFP) & 306 & 218 & 3124 & $.07(.06-.08)$ & 203 & $\mathrm{~N}$ & - & - \\
\hline Ciprofloxacin (CIP) & 714 & 490 & 6552 & $.07(.06-.08)$ & 455 & $\mathrm{~N}$ & - & - \\
\hline Levofloxacin (Lvx) & 73 & 48 & 652 & $.07(.06-.1)$ & 48 & $\mathrm{~N}$ & - & - \\
\hline Colistin (CST) & 87 & 13 & 934 & $.01(.008-.02)$ & 11 & $\mathrm{~N}$ & - & - \\
\hline Imipenem (IPM) & 563 & 145 & 5517 & $.02(.02-.03)$ & 147 & $\mathrm{~N}$ & - & - \\
\hline Meropenem (MEM) & 319 & 95 & 3285 & $.029(.023-.035)$ & 114 & $\mathrm{~N}$ & - & - \\
\hline Cotrimoxazole(SXT) & 530 & 410 & 4911 & $.08(.07-.09)$ & 351 & $Y$ & .1 & 395 \\
\hline
\end{tabular}

${ }^{1}$ Significant difference was not observed

${ }^{2}$ Signficant difference was observed 
Table 5: Incidence rate and optimized incidence rate of Antimicrobials Resistance E.coli in <q1 quarter

\begin{tabular}{|c|c|c|c|c|c|c|c|}
\hline $\begin{array}{l}\text { Antimicrobial } \\
\text { agents }\end{array}$ & sus & obs & $\begin{array}{c}\text { Incidence rate } \\
(95 \% \mathrm{CI})\end{array}$ & $\exp$ & $\begin{array}{l}\text { Significant } \\
\text { difference }\end{array}$ & $\begin{array}{c}\text { Optimized } \\
\text { Incidence rate }\end{array}$ & $\begin{array}{c}\text { Optimized } \\
\text { expected cases }\end{array}$ \\
\hline Ampicillin (AMP) & 56 & 44 & $.4(.3-.5)$ & 36 & $\mathrm{~N}^{1}$ & - & - \\
\hline Ceftazidime (CAZ) & 150 & 82 & $.28(.18-.38)$ & 72 & $\mathrm{~N}$ & - & - \\
\hline Ceftriaxone (CEF) & 213 & 140 & $.42(.35-.50)$ & 117 & $\mathrm{Y}^{2}$ & .42 & 141 \\
\hline Cefotaxime (CTX) & 158 & 110 & $.45(.35-.55)$ & 91 & $\mathrm{Y}$ & .45 & 110 \\
\hline Cefepime (CFP) & 114 & 69 & $.3(.2-.4)$ & 58 & $\mathrm{~N}$ & - & - \\
\hline Ciprofloxacin (CIP) & 342 & 216 & $.4(.3-.5)$ & 175 & $\mathrm{Y}$ & .4 & 218 \\
\hline Levofloxacin (Lvx) & 31 & 20 & $.6(.5-.8)$ & 19 & $\mathrm{~N}$ & - & - \\
\hline Colistin (CST) & 29 & 7 & $.08(.04-.12)$ & 6 & $\mathrm{~N}$ & - & - \\
\hline Imipenem (IPM) & 223 & 61 & $.14(.11-.18)$ & 58 & $\mathrm{~N}$ & - & - \\
\hline Meropenem (MEM) & 108 & 29 & $.14(.12-.18)$ & 28 & $\mathrm{~N}$ & - & - \\
\hline Cotrimoxazole(SXT) & 257 & 185 & $.47(.36-.57)$ & 152 & $\mathrm{Y}$ & .47 & 185 \\
\hline
\end{tabular}

1Significant difference was not observed

${ }^{2}$ Signficant difference was observed 
Table 6: characteristics of the developed model based on the antibiotics

\begin{tabular}{|c|c|c|c|c|c|c|c|c|}
\hline $\begin{array}{l}\text { Antimicrobial } \\
\text { agents }\end{array}$ & \multicolumn{2}{|c|}{ Ir } & \multicolumn{2}{|c|}{$\mathrm{rr}^{\mathbf{b}}$} & \multicolumn{2}{|c|}{$D^{d}$} & \multicolumn{2}{|c|}{ START TIME- STOP TIME } \\
\hline Ampicillin (AMP) & .08 & .4 & .1 & .1 & 1 & 1 & $4-20$ & $2-4$ \\
\hline Ceftazidime (CAZ) & .07 & .28 & .09 & .09 & 1 & 1 & $4-18$ & $2-4$ \\
\hline Ceftriaxone (CEF) & .08 & .33 & .1 & .1 & 1 & 1 & $4-18$ & $2-4$ \\
\hline Cefepime (CFP) & .07 & .3 & .08 & .08 & 1 & 1 & $4-19$ & $2-4$ \\
\hline Ciprofloxacin (CIP) & .07 & .3 & .1 & .1 & 1 & 1 & $4-18$ & $2-4$ \\
\hline Levofloxacin (Lvx) & .07 & .6 & .14 & .14 & 1 & 1 & $3-18$ & $2-3$ \\
\hline Meropenem (MEM) & .029 & .14 & .09 & .09 & 1 & 1 & $4-19$ & $2-4$ \\
\hline Cotrimoxazole(SXT) & .08 & .36 & .11 & .11 & 1 & 1 & $4-17$ & $2-4$ \\
\hline
\end{tabular}

\footnotetext{
e: optimized incidence rate

b: remove rate per day

$\mathrm{d}$ : size of time step in daily units

${ }^{\circ}$ : time period over which the model is run in daily units
} 
Table 7: characteristics of the developed and optimized model based on the antibiotics

\begin{tabular}{|c|c|c|c|c|c|c|c|c|}
\hline $\begin{array}{l}\text { Antimicrobial } \\
\text { agents }\end{array}$ & \multicolumn{2}{|c|}{ Ir } & \multicolumn{2}{|c|}{$\mathbf{r r}^{\mathbf{b}}$} & \multicolumn{2}{|c|}{$\mathrm{DT}^{\mathrm{d}}$} & \multicolumn{2}{|c|}{ START TIME- STOP TIME } \\
\hline Ampicillin (AMP) & .08 & .4 & .1 & .1 & 1 & 1 & $4-20$ & $2-4$ \\
\hline Ceftazidime (CAZ) & .07 & .28 & .09 & .09 & 1 & 1 & $4-18$ & $2-4$ \\
\hline Ceftriaxone (CEF) & $.1^{\mathrm{e}}$ & $.42^{\mathrm{e}}$ & .1 & .1 & 1 & 1 & $4-18$ & $2-4$ \\
\hline Cefepime (CFP) & .07 & .30 & .08 & .08 & 1 & 1 & $4-19$ & $2-4$ \\
\hline Ciprofloxacin (CIP) & .07 & $.4^{\mathrm{e}}$ & .1 & .1 & 1 & 1 & $4-18$ & $2-4$ \\
\hline Levofloxacin (Lvx) & .07 & .6 & .14 & .14 & 1 & 1 & 3-18 & $2-3$ \\
\hline Colistin (CST) & .01 & .08 & .06 & .06 & 1 & 1 & $5-18$ & $2-5$ \\
\hline Cotrimoxazole(SXT) & $.1^{\mathrm{a}}$ & $.47^{\mathrm{e}}$ & .11 & .11 & 1 & 1 & $4-17$ & $2-4$ \\
\hline
\end{tabular}

e: optimized incidence rate

b: remove rate per day

d: size of time step in daily units

${ }^{\text {ə: }}$ : time period over which the model is run in daily units 
Table 8: calculation of SIR index based on antibiotic

\begin{tabular}{|c|c|c|c|c|c|c|c|}
\hline \multirow{2}{*}{$\begin{array}{l}\text { Antimicrobial } \\
\text { agents }\end{array}$} & \multirow[t]{2}{*}{ range } & \multirow[t]{2}{*}{ sus } & \multirow[t]{2}{*}{ obs } & \multirow[t]{2}{*}{$\exp$} & \multirow[t]{2}{*}{ SIR } & \multicolumn{2}{|c|}{$95 \% \mathrm{Cl}$} \\
\hline & & & & & & Lower & Upper \\
\hline \multirow[t]{2}{*}{ Ampicillin (AMP) } & Q1-Q3 & 215 & 192 & 158 & 1.2 & 1.1 & 1.3 \\
\hline & Q1> & 87 & 74 & 56 & 1.3 & 1.1 & 1.6 \\
\hline \multirow[t]{2}{*}{ Ceftazidime (CAZ) } & Q1-Q3 & 568 & 399 & 362 & 1.1 & 1.02 & 1.2 \\
\hline & Q1> & 241 & 142 & 116 & 1.2 & 1.03 & 1.4 \\
\hline \multirow[t]{2}{*}{ Ceftriaxone (CEF) } & Q1-Q3 & 575 & 429 & 443 & 1.03 & .96 & 1.1 \\
\hline & Q1> & 256 & 183 & 170 & 1.08 & .96 & 1.2 \\
\hline \multirow[t]{2}{*}{ Cefotaxime (CTX) } & Q1-Q3 & 525 & 417 & 405 & 1.03 & 1 & 1.1 \\
\hline & Q1> & 208 & 149 & 145 & 1.03 & .90 & 1.2 \\
\hline \multirow[t]{2}{*}{ Cefepime (CFP) } & Q1-Q3 & 338 & 228 & 224 & 1.01 & .90 & 1.1 \\
\hline & Q1> & 125 & 72 & 63 & 1.1 & .90 & 1.4 \\
\hline \multirow[t]{2}{*}{ Ciprofloxacin (CIP) } & Q1-Q3 & 802 & 537 & 512 & 1.05 & .98 & 1.1 \\
\hline & Q1> & 413 & 267 & 264 & 1.01 & .90 & 1.1 \\
\hline \multirow[t]{2}{*}{ Levofloxacin (Lvx) } & Q1-Q3 & 122 & 82 & 81 & 1 & .80 & 1.2 \\
\hline & Q1> & 41 & 27 & 24 & 1.12 & .80 & 1.6 \\
\hline \multirow[t]{2}{*}{ Colistin (CST) } & Q1-Q3 & 151 & 31 & 18 & 1.7 & 1.02 & 2.9 \\
\hline & Q1> & 78 & 10 & 17 & .60 & .30 & 1.2 \\
\hline \multirow[t]{2}{*}{ Imipenem (IPM) } & Q1-Q3 & 761 & 174 & 199 & .87 & .73 & 1.1 \\
\hline & Q1> & 341 & 53 & 89 & .60 & .40 & .80 \\
\hline \multirow[t]{2}{*}{ Meropenem (MEM) } & Q1-Q3 & 389 & 110 & 138 & .8 & .65 & .98 \\
\hline & Q1> & 156 & 27 & 41 & .7 & .4 & 1 \\
\hline
\end{tabular}




\begin{tabular}{|l|l|l|l|l|l|ll|}
\hline Cotrimoxazole(SXT) & Q1-Q3 & 578 & 433 & 431 & 1 & .9 & 1.1 \\
\cline { 2 - 7 } & Q1> & 283 & 205 & 203 & 1 & .9 & 1.1 \\
\hline
\end{tabular}

\section{Discussion}

In order to assess selection bias, we compared individuals who were included in the study regarding demographic variables (age and gender) with those who were not included in the study. Mean age of those who were included in the study and those who were not included was $57(\mathrm{SE}=0.4)$ and 58 (0.7), respectively. 55\% of those entered the study were man. This was $48 \%$ for those who were not entered. There was no considerable difference among these two groups regarding the variables which can indicate the representativeness of the study.

AMP-resistant E.coli observed cases were 20\% greater than the expected cases, which this difference is significant. The results of this part of the study were consistent with the results of the other part of the study (observed cases were significantly $10 \%$ higher than expected). This could mean that the incidence of ampicillin-resistant E.coli has increased significantly, which is consistent with studies conducted in Turkey, Iran and Ireland (1719).

CAZ-resistant E.coli observed cases were 10\% greater than the expected cases, which this difference was also significant. The results of this part of the study were consistent with the results of the other part of the study (observed cases were $20 \%$ greater than the expected ones, which was also significant in this part). In other words, it can be stated that in both time periods, an outbreak of ceftazidium-resistant E.coli occurred. The results of this section were inconsistent with a study conducted in China (20). This may be due to differences in the years of the two studies.

CEF-resistant E.coli observed cases were 3\% greater than the expected ones, and this difference was not significant. The results of this part of the study were consistent with the results of the other part of the study (the Ceftriaxone resistant observed cases were 8\% 
greater than the expected ones, which this difference was not significant). In other words, in both sections, there was no outbreak and the observed cases were as expected. This may be due to the proper use of antibiotics in the treatment of these infections. The results of this part of the study are consistent with a study conducted in China and Turkey $(17,20)$. But it did not correspond to a study conducted in Indonesia(21).

CTX-resistant E.coli observed cases were 3\% greater than the expected cases, which this difference was not significant. The results of this part of the study were consistent with the results of the other part of the study (observed cases were 3\% greater than the expected cases, which this difference was also not significant). This may be due to the proper use of antibiotics in the treatment of these infections. The results of this part of the study were consistent with a study conducted in China, but did not match the study in Indonesia (20, 21).

Observed cases of CFP-resistant E.coli were 1\% greater than the expected cases, which this difference was not significant. The results of this part of the study were consistent with the results of the other part of the study (observed cases of Cefepime resistance was 10\% greater than the expected cases, which this difference was also not significant). Therefore, there was no outbreak in both sections and incidence of this infection has not changed significantly. This may be due to the proper use of antibiotics in the treatment of these infections. The results of this part of the study were consistent with a study conducted in China(21).

Observed cases of CIP-resistant E.coli were 5\% greater than the expected cases, which this difference was not significant. The results of this part of the study were consistent with the results of the other part of the study (observed cases of Ciprofloxacin resistance was 1\% greater than the expected cases, which this difference was also not significant). The results of this part of the study were consistent with a study conducted in China, but did not match the study in Turkey $(17,21)$

Observed cases of lvx-resistant E.coli were the same as the expected cases. The results of this part of the study were consistent with the results of the other part of the study (observed cases of resistance was 12\% greater than the expected cases, but this difference was not significant). Therefore, there was no outbreak in both sections and incidence of this infection has not changed significantly. This may be due to the proper use of antibiotics in the treatment of these infections. The results of this part of the study were consistent with a study conducted in China (20). These antibiotics, along with ciprofloxacin, belong to the class of fluoroquinolones. This study showed that fluoroquinolone-resistant cases were seen as expected and the incidence of antibiotic-resistant cases was somewhat unchanged.

Observed cases of CST-resistant E.coli were 70\% greater than the expected cases, which this difference was also significant. The results of this part of the study were not consistent with the other part (observed cases of resistance was $40 \%$ lesser than the expected cases, which this difference was significant). In the two sections studied, the SIR is completely different and do not match. Due to the high cost of antibiogram testing for Colistin and also low incidence of this type of resistance, so, it seems that more tests are needed for 
comparison between observed and expected cases. Hence, we can not talk about the results of this section with certainty.

Observed cases of IPM-resistant E.coli were 13\% lesser than the expected cases, which this difference was not significant. The results of this part of the study were consistent with the results of the other part of the study (observed cases of Imipenem resistance was $40 \%$ lesser than the expected cases, which this difference was significant). In other words, in this study, for reasons such as improving health conditions, the good performance of this antibiotic against these infections, was as expected and better than expected. In addition, observed cases of MEM-resistant E.coli were 20\% lesser than the expected cases, which this difference was also significant. The results of this part of the study were consistent with the results of the other part of the study (observed cases of Meropenem resistance was 30\% lesser than the expected cases). In other words, it can be said that the action of Meropenem against nosocomial infections caused by E.coli was also appropriate. The results of this part of the study were consistent with a study conducted in Iran and Ugandan $(18,22,23)$.

Observed cases of SXT-resistant E.coli were the same as the expected cases. The results of this part of the study were consistent with the results of the other part of the study (observed cases of resistance was not considerably different from the expected cases). The results of this part of the study were consistent with a study conducted in China (20).

\section{Conclusions}

Due to the results of this study, at 2017, among the nosocomial infections caused by E.coli microbe, Emerging was occurred in AMP-resistant E.coli and CAZ -resistant E.coli. Hence, it is necessary to reconsider using such type of antibiotics in treatment of nosocomial infections caused by E.coli.

Using the method used in this study, it is possible to assess the outbreak of resistant E.coli at longer or shorter intervals, such as the year or month. This method can also be used at the hospital level to monitor the outbreak of these infections. Also in the coming years with the help of indicators estimated by this method the trend of these types of infections can be examined. The results of this study could be important for health policy makers. 


\section{References}

1. World Health Organization. (2014). Antimicrobial resistance: global report on surveillance. World Health Organization. https://apps.who.int/iris/handle/10665/112642

2. Theuretzbacher U. Global antibacterial resistance: The never-ending story. Journal of global antimicrobial resistance. 2013; 1(2):63-9. https://doi.org/10.1016/j.jgar.2013.03.010

3. Ventola CL. The antibiotic resistance crisis: part 1: causes and threats. P \& $T$ : a peer-reviewed journal for formulary management. 2015;40(4):277-83.

4. FDAmini M, Vaseie M, Ansari I. THE EVALUATION OF NOSOCOMIAL URINARY TRACT INFECTIONS AND ANTIMICROBIAL RESISTANCE IN ICU PATIENTS, TEHRAN, IRAN, 2012-2016. Acta Medica Mediterr. 2017;33:945-52.

5. Peirano G, Pitout JD. Molecular epidemiology of Escherichia coli producing CTX-M betalactamases: the worldwide emergence of clone ST131 O25:H4. International journal of antimicrobial agents. 2010;35(4):316-21.

6. Johnson JR, Johnston B, Clabots C, Kuskowski MA, Castanheira M. Escherichia coli sequence type ST131 as the major cause of serious multidrug-resistant E. coli infections in the United States. Clinical infectious diseases : an official publication of the Infectious Diseases Society of America. 2010;51(3):28694.

7. Johnson JR, Menard M, Johnston B, Kuskowski MA, Nichol K, Zhanel GG. Epidemic clonal groups of Escherichia coli as a cause of antimicrobial-resistant urinary tract infections in Canada, 2002 to 2004. Antimicrob Agents Chemother. 2009;53(7):2733-9.

8. de Kraker ME, Davey PG, Grundmann H. Mortality and hospital stay associated with resistant Staphylococcus aureus and Escherichia coli bacteremia: estimating the burden of antibiotic resistance in Europe. PLoS medicine. 2011;8(10):e1001104.

9. Global Action Plan on Antimicrobial Resistance [cited 2015 Available from: http://apps.who.int/gb/ebwha/pdf files/WHA68/A68 20-en.pdf?ua=1.

10. World Health Organization (WHO). Global Antimicrobial Resistance Surveillance System: Manual for Early Implementation. http://www.who.int/antimicrobial-resistance/publications/surveillancesystem-manual/en/. ISBN: 9789241549400. Geneva, Switzerland; 2016. p. 1-4.

11. Masumi Asl H, National Guideline of Nosocomial Infections Surveillance. Tehran: Markaz Modiriat Bimariha.2016. ISBN: 964 -6570-65-8.

12. CDC. THE NHSN STANDARDIZED INFECTION RATIO (SIR) 2018 [Available from: https://www.cdc.gov/nhsn/pdfs/ps-analysis.../nhsn-sir-guide.

13. Tacconelli E, Cataldo MA, Paul M, Leibovici L, Kluytmans J, Schr H\|der W, et al. STROBE-AMS: recommendations to optimise reporting of epidemiological studies on antimicrobial resistance and informing improvement in antimicrobial stewardship. BMJ Open. 2016;6(2):e010134.

14. Moons KG, Altman DG, Reitsma JB, loannidis JP, Macaskill P, Steyerberg EW, et al. Transparent Reporting of a multivariable prediction model for Individual Prognosis or Diagnosis (TRIPOD): explanation and elaboration. Annals of internal medicine. 2015;162(1):W1-73.

15. E. Vynnycky, White RG. An Introduction to Infectious Disease Modelling. NY, New York: Oxford University Press; 20102010. 
16. Szklo M, Nieto FJ. Epidemiology : beyond the basics2019.

17. A, S. B., Ciftci IH. [Antibiotic resistance patterns of Escherichia coli strains isolated from urine cultures in Turkey: a meta-analysis]. Mikrobiyol Bul. 2013;47(4):603-18.

18. DGhadiri $\mathrm{H}$, Vaez $\mathrm{H}$, Khosravi S, Soleymani $\mathrm{E}$. The antibiotic resistance profiles of bacterial strains isolated from patients with hospital-acquired bloodstream and urinary tract infections. Critical care research and practice. 2012;2012.

19. Gohel K, Jojera A, Soni S, Gang S, Sabnis R, Desai M. Bacteriological profile and drug resistance patterns of blood culture isolates in a tertiary care nephrourology teaching institute. Biomed Res Int. 2014;2014.

20. Li XY, Chen YQ, Gao WG, Ye H, Shen ZC, Wen ZH, et al. A 6-year study of complicated urinary tract infections in southern China: prevalence, antibiotic resistance, clinical and economic outcomes. Therap Clin Risk Manag. 2017;13:1479-87.

21. [Lo WT, Lin WJ, Chiueh TS, Lee SY, Wang CC, Lu JJ. Changing trends in antimicrobial resistance of major bacterial pathogens, 1985-2005: A study from a medical center in northern Taiwan. Journal of Microbiology Immunology and Infection. 2011;44(2):131-8.

22. Al-Hasan MN, Lahr BD, Eckel-Passow JE, Baddour LM. Antimicrobial resistance trends of Escherichia coli bloodstream isolates: a population-based study, 1998-2007. J Antimicrob Chemother. 2009;64(1):169-74.

23. Agaba P, Tumukunde J, Tindimwebwa JVB, Kwizera A. Nosocomial bacterial infections and their antimicrobial susceptibility patterns among patients in Ugandan intensive care units: a cross sectional study. BMC research notes. 2017;10(1):349. 